\title{
LED lighting. Creation and application peculiarities
}

\author{
Yu.V. Trofimov, S.I. Lishik, V.S. Posedko, V.I. Tsvirko, A.A. Pautino \\ The Republican Scientific and Production Unitary Enterprise "Center of LED and optoelectronic technologies of \\ National Academy of Sciences of Belarus", \\ 22/2207, Logoiski Trakt, Minsk BY-220090, The Republic of Belarus
}

\begin{abstract}
State-of-the-art condition of LED illumination engineering for street lighting, its requirements, construction technological features of its creation, optimization ways of optical, thermal and electrical luminaire subsystems are considered in this article. Besides, the results of own researches and development in the field of outside lighting are reported here.
\end{abstract}

Keywords: LED lighting, LED street light, outside lighting.

Manuscript received 18.12.09; accepted for publication 25.03.10; published online 30.04.10.

\section{Introduction}

The growth of the world population and global economy results in increasing demands for electric power, which is satisfied by new generating capacities all over the world. Therewith the fast exhaustion of minerals (in the first place, oil, gas and nuclear fuel) and environmental pollution is taking place. The only way out of this situation seems to be developing and using alternative energy sources (wind, sun, geothermal sources, tides, etc.), as well as resource and energy saving.

Lighting is one of promising implementation fields of resource and energy saving technologies that are caused by scientific and technological progress in creating high-performance solid-state light sources (light-emitting diodes). Indeed, if to take for example Belarus, a share in electric power consumed on lighting is about $18 \%$. Therefore, considering energy consumption volume in Belarus (35 bn $\left.\mathrm{kW} \cdot \mathrm{h}\left[{ }^{1}\right]\right)$, we have some $6.3 \mathrm{bn} \mathrm{kW} \cdot \mathrm{h}$ consumed on lighting needs. From the financial point of view, it means that about $\$ 630 \mathrm{mln}$ (at the average power rate $\$ 0.1$ per $\mathrm{kW} \cdot \mathrm{h}$ ) turns into light annually, and in relation to ecology, some $4 \mathrm{mln}$ tons of carbon dioxide $\mathrm{CO}_{2}$ are escaped into environment. For instance, lighting costs are $\$ 2$ to 3 bn per year in Ukraine, and \$ 9 to 10 bn per year in Russia.

A huge volume of finance and electrical power wasted annually on lighting needs is partly caused by a low performance of the light sources presently used, mainly incandescent lamps. Light-emitting diode is an alternative to traditional light source and is characterized by the following advantages: high luminous efficiency (at present about $100 \mathrm{~lm} / \mathrm{W}$ for commercially available LEDs), long-term service (50000 to 100000 hours), acceptable light quality (color rendering index $>75$ ), ecological compatibility (lack of mercury components), vibration stability (due to lack of moving mechanical portions), electrical safety (due to low supply voltage). Due to features mentioned above, DOE experts (USA) predict replacement of traditional light sources by LED sources in the nearest future. For that, a number of countries have passed the laws prohibiting the use of incandescent lamps: Great Britain (since 2009), California State in the USA (by 2012), Canada (since 2012), Australia (since 2010), EU (since 2012), the Ukraine (since 1 January 2009) and Russia (since 2010). Apart from statutory bar for the use of incandescent lamps there is unconstrained refusal from their production. For instance, European manufacturers of lighting equipment initiated to phase out the production of low-performance incandescent lamps by 2015 .

Among a great variety of different LED facilities, LED street lights can be distinguished, which is caused by a major portion of electric power consumed by outside lighting. The prospectivity of products in this field is also proved by an increased number of patent documents issues on LED street lights (Fig. 1).

That is why this work is aimed at considering construction technological features of LED street lights. 


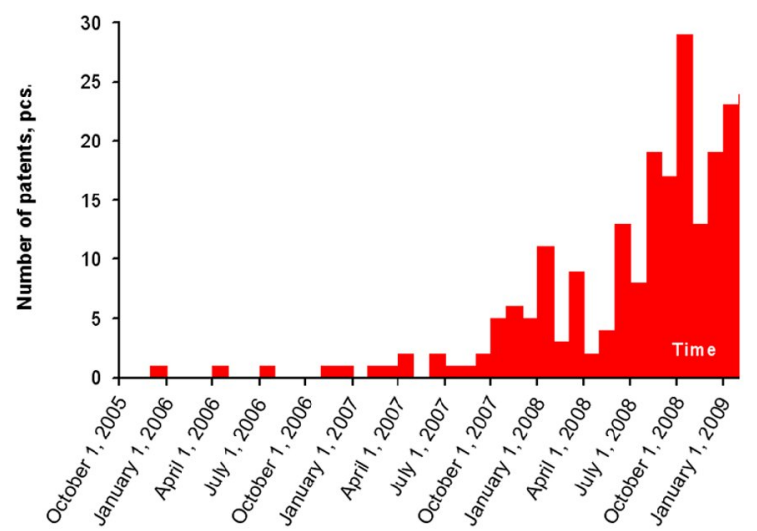

Fig. 1. Time dependence of the number of patent document issues on LED street lights.

\section{LED light subsystems}

As it is generally known, thermal, optical and electrical subsystems are main subsystems of any LED luminaire (not only street light). Efficiency of each subsystem can be described in terms of quantity with factors $\eta_{\text {therm }}$, $\eta_{\text {opt }}$ and $\eta_{\mathrm{el}}$ (Table 1), $\eta_{\text {therm }}$ - efficiency factor of the thermal subsystem, $\eta_{\text {opt }}$ - efficiency factor of the optical subsystem, $\eta_{\mathrm{el}}$ - efficiency factor of power supply/control unit;

Table 1. Ranges of loss factors values, typical for products of different performance level.

\begin{tabular}{|l|l|l|l|}
\hline$\eta_{\text {opt }}$ & $\eta_{\text {el }}$ & $\eta_{\text {therm }}$ & $\eta$ \\
\hline $0.50 \ldots 0.92$ & $0.80 \ldots 0.97$ & $0.85 \ldots 0.98$ & $0.34 \ldots 0.87$ \\
\hline
\end{tabular}

The total luminaire efficiency $\eta$ is defined by multiplication of the factors in accordance to the formula:

$\eta=\eta_{\text {therm }} \cdot \eta_{\text {opt }} \cdot \eta_{\text {el }}$,

It is easy to see that luminaire efficiency $\eta$ lies within the wide range from 0.34 to 0.87 . Therefore, the task of LED light developers is maximum possible approximation to the upper edge of luminaire efficiency (0.87). The task is complicated but rather achievable. Thereto it is necessary to optimize separately the thermal, optical and electrical subsystems of luminary. Let us see how to solve these tasks in practice.

\subsection{Thermal design}

The value of the thermal loss factor $\eta_{\text {therm }}$ is defined by decreasing luminous flux $\Phi$ at increasing LED operating temperature (Fig. 2)

$\eta_{\text {therm }}=\frac{\Phi\left(T_{j}\right)}{\Phi\left(T_{\mathrm{amb}}\right)}$

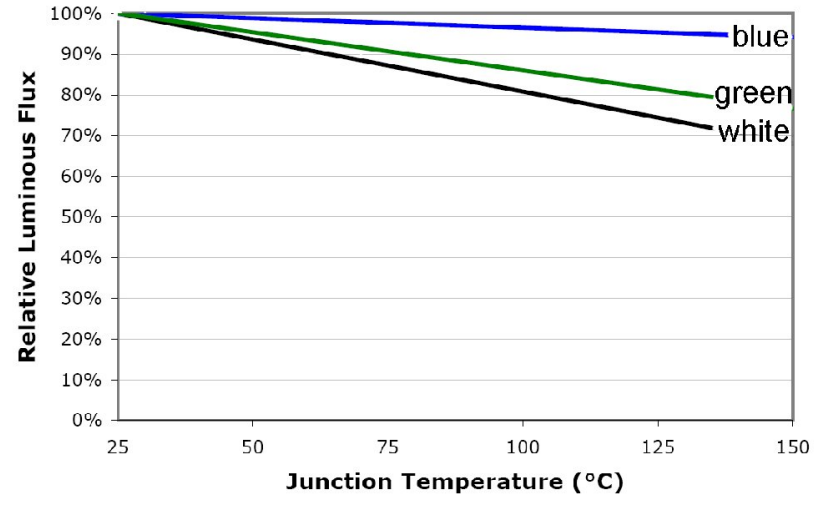

Fig. 2. Characteristic dependence of normalized luminous flux on temperature for LEDs XR-E series, produced by CREE company.

where $T_{j}$ is the operating temperature of LED active region, $T_{\mathrm{amb}}$ - ambient temperature.

Therefore, the less the temperature difference $T_{j}-T_{\mathrm{amb}}=\Delta T$, the bigger thermal subsystem efficiency factor $\eta_{\text {therm }}$.

As $\Delta T$ is connected with thermal resistance by the ratio:

$\Delta T=R_{\theta} P$,

where $R_{\theta}$ is the thermal resistance between LED active region and environment; $P-$ part of power consumption, spread as heat, in this case thermal design optimization task brings to the minimization of all thermal interfaces between LEDs and environment, i.e. decreasing the LED active region temperature, and providing identical temperature conditions for all lightemitting diodes.

The use of cases with large heat-dissipating square, provided by die casting, or the use of extrusion profile packages is included in typical optimization method of thermal design of LED street light (Fig. 3). Therewith, considering minimization of LED product cost, the second way is preferable.

Holing the fins of a radiator, thus enabling to increase heat spreading factor, is an additional optimization way (apart from mentioned) of the thermal subsystem. Besides, aluminum, copper and glass textolite (FR-4) with thermal via printed circuit boards, as well as heat-conducting glues, pastes, etc. are applied.

The decrease of temperature in the LED active region, which increases significantly its term of service, is the major advantage of such an approach. For instance, calculations in [2] show that the use of heat pipes makes it possible to decrease the temperature of LED active region by $5-10{ }^{\circ} \mathrm{C}$ and even more, that is equivalent to increasing LED term of service by $15-18$ thousand hours, as well as luminous flux increasing by 10-20 per cent or increasing the upper edge of the operation temperature range. 


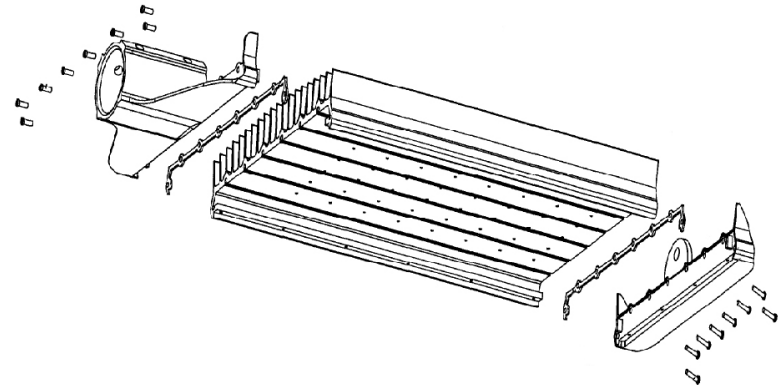

Fig. 3. Optimization of the thermal design of LED street lights using extrusion profile packages.

The use of heat pipes is another way of optimization of the thermal design (Fig. 4).

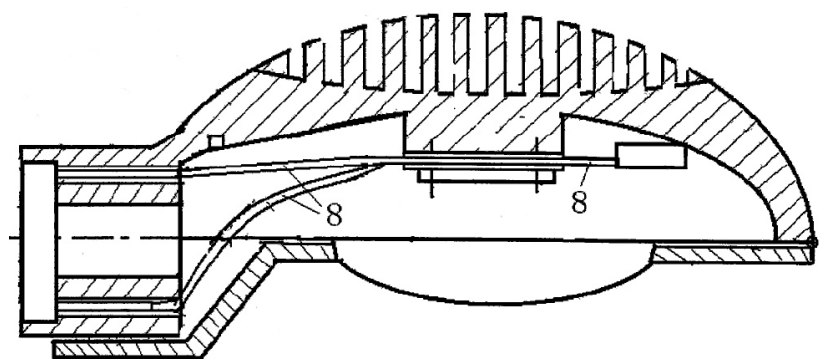

Fig. 4. Optimization of the thermal design of LED street lights using heat pipes.

Besides, the use of heat pipes enables to align the temperature of light-emitting diodes and to lighten the luminaire, because there is no need in concentrating much heavy metal nearby light-emitting diodes to take away the heat.

Covering the thermal radiator with the shroud with a lot of air holes is one more widespread way of the optimization of the thermal design (Fig. 5).

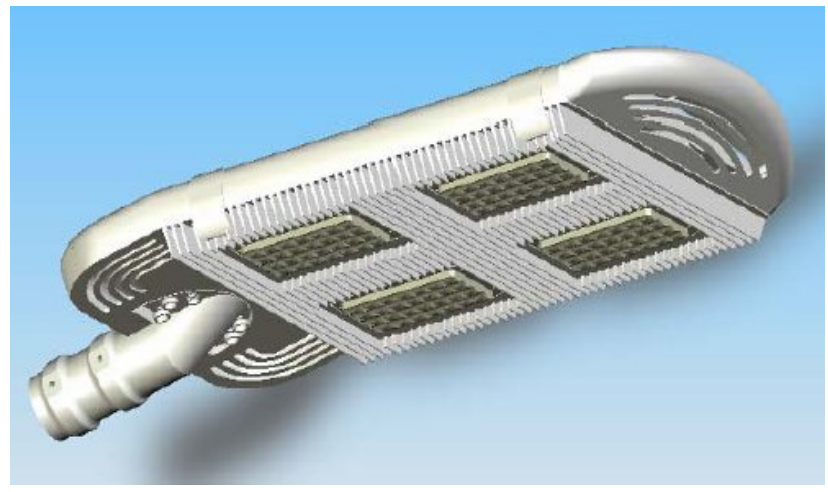

Fig. 5. Optimization of the thermal design of LED street lights by air holes.
Due to pressure changes between the thermal radiator and the housing of luminaire in the groove there is an air draught, and thus the heat dissipating capacity of the radiator is improved. Besides, the probability of the thermal radiator dusting is going down; the radiator is better corrosion-protected, and light-emitting diodes are heat-protected during a bright shiny day due to use of radiator (that is particularly urgent for the southern climate when the luminaire package can be heated up to $\left.120{ }^{\circ} \mathrm{C}\right)$.

Among the imperfections of this approach is the probability of organic and inorganic dusting at the bottom and sideways, though not so large as in case of the thermal radiator that is not covered with the housing. To reduce the inner cavity of luminaire dusting through air holes, it is necessary to optimize their size and shape: they must be very wide to promote air flowing, as well as rather narrow to prevent quite big objects (e.g. leaves) from penetrating into the luminaire.

\subsection{Optical design}

Now look at the optical subsystem of LED street light.

Four main requirements for the optical subsystem of LED street lights can be defined as follows:

- high efficiency $\eta_{\text {opt }}$;

- standardized average level of illuminance;

- high homogeneity and uniformity of illuminance;

- rectangular geometry of illuminated square.

Let's consider them in detail.

The efficiency of optical luminaire subsystem depends on optical losses, accuracy of manufacturing the optical elements (reflector, diffusers, lenses, shade) as well as quality of used materials (polycarbonate glass, aluminum reflectors).

The code of practice 23-05-95 (Russia) includes recommended levels of illuminance and illuminance non-uniformity factors for highways of different category. According to this document highways can be divided into three categories: A-category - 15 to 20 lux, B-category -10 to 15 lux, C-category -4 to 6 lux. These illuminance level standards have been developed according to the spectral characteristics of traditional light sources and nowadays they have gone out of date. In particular, if to compare different sensations of using luminaries with the same level of luminous flux, based on high-pressure sodium lamps and light-emitting diodes, the LED light seems to be more luminous. In fact, it is not the case; the reason is that human eye differently perceives radiation depends on light conditions and correlated color temperature. Human eyesight mostly reacts to wavelength $550-555 \mathrm{~nm}$ (photopic view) in the daytime, and approximately $507 \mathrm{~nm}$ (scotopic view) in the darkness. High-pressure sodium lamps have practically zero radiation in the green area (500-550 nm), while LED luminaries have quite intensive radiation in this part. Besides, LED light is characterized by higher value of color temperature (CCT) in comparison with high-pressure sodium lamps, 
and therefore it is subjectively perceived as more luminous $\left[{ }^{3}\right]$. Therefore, illuminance level requirements in relation to LED street lights can be reduced. According to some companies, for instance BetaLED (USA) $\left[{ }^{4}\right]$, it is reasonable to use step-down factor 0.4 for illuminance level requirements based on highpressure sodium lamps for LED light systems.

In most cases, the illuminated square has a squared shape (e.g. ways, the pedestrian crossing, bridges, etc.). Therefore, the task for developed illuminator is to light up the motorway with the most intensity and adjacent area with the less intensity. For this purpose luminous intensity distribution curve should be as shown in Fig. 6.

Developers' efforts are concentrated mostly on elaboration ways of changing the luminous intensity distribution curve. The following approaches are used here:

- LED printed circuit boards are placed under different angles to each other;

- light-emitting diodes are used together with special elements of secondary optics (reflectors, lenses and LEDs);

Approach to design of LED street lights, based on LEDs placing under different angles to each other (Fig. 7), enables to get the required diagram of light output, though it requires to fulfill a lot of manual operations during the luminaries assembly (for instance, connection of semiconductors, board assembly and deformation), that significantly raises the luminaire cost. Therefore, this approach is not possible when LED street lights are designed for mass production.

Reflectors can be divided into the following types (Fig. 8):

- reflectors-concentrators of radiation (Fig. 8a), which enable to increase the level of illuminance, and type of luminous intensity distribution curve is not changed. The drawback of this type of reflectors is a small area of illuminated square. Nevertheless, luminaries with such kind of reflectors can be required, for instance, for lighting of squares;

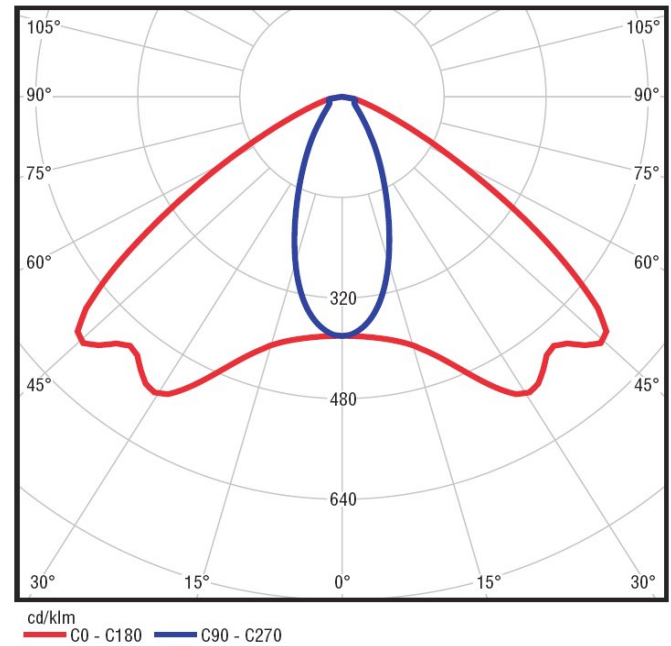

Fig. 6. Light diagram for LED street light.
- reflectors which modify the diagram of light output (Fig. 8b). One couple of squares of this kind reflectors compresses the diagram of light output in crosswise direction, and the other one extends in lengthwise direction;

- reflectors in the shape of V-grooves (Fig. 8c). There are two kinds of these reflectors which are known today: with positive and negative angles. It should be noticed that it is technologically complicated to manufacture reflectors with negative angles.

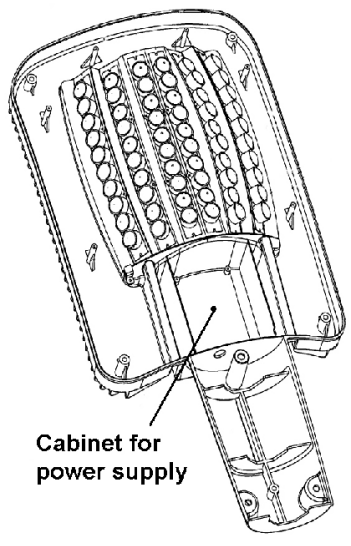

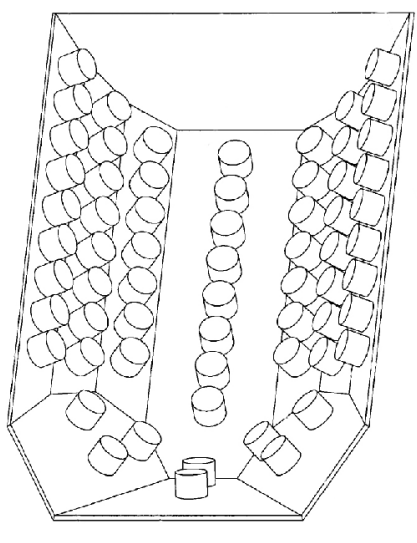

b
Fig. 7. Creation of the necessary light diagram for LED street light by placing LEDs under different angles to each other.

a

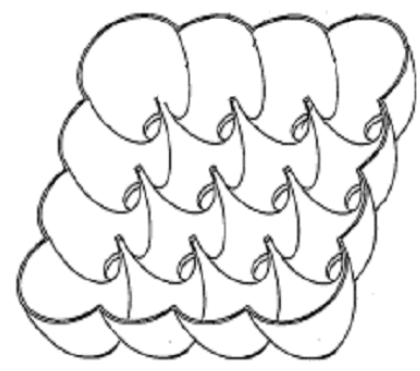

$\mathrm{b}$

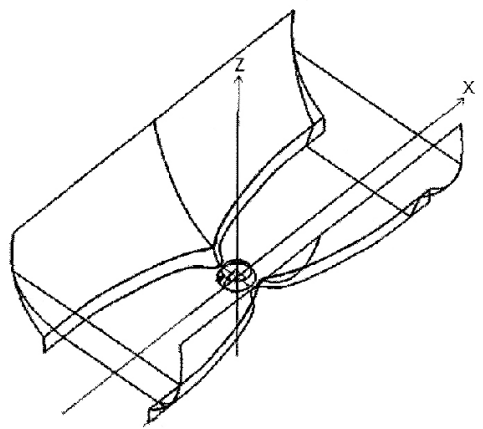

c

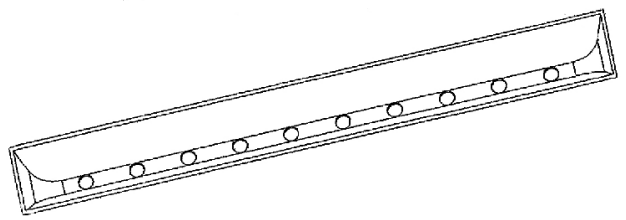

Fig. 8. Types of reflectors for modifying LED luminous intensity distribution curves. 
And now we consider some approaches to manufacturing lenses of secondary optics for LED street lights (Fig. 9).

We can mark out the following lenses:

- lenses based on effect of total internal reflection (Fig. 9a,b). This lens deviates the direction of maximum LED light output to a definite angle;

- lenses which consist of two fragments of spherical squares connected by bridge (Fig. 9c,d). For instance, these lenses could be of STRADA-B-GD series for light-emitting diodes Platinum/Dragon+ of Osram Opto company;

- "collective" lenses (Fig. 9e).

a

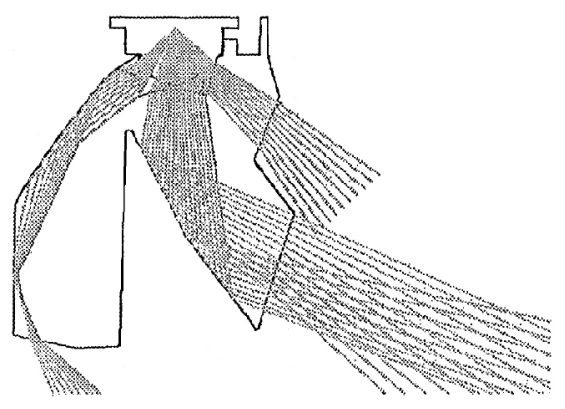

b

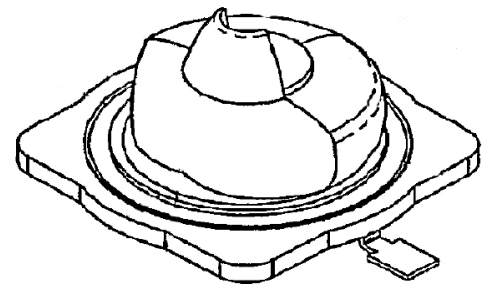

$\mathrm{c}$

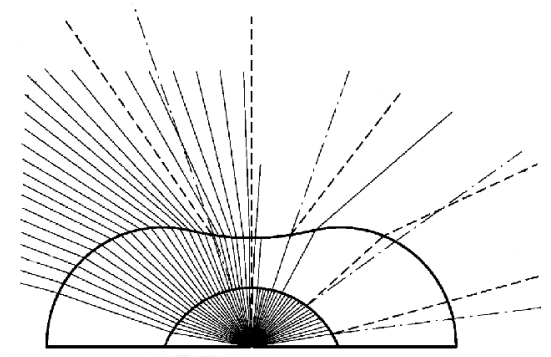

d
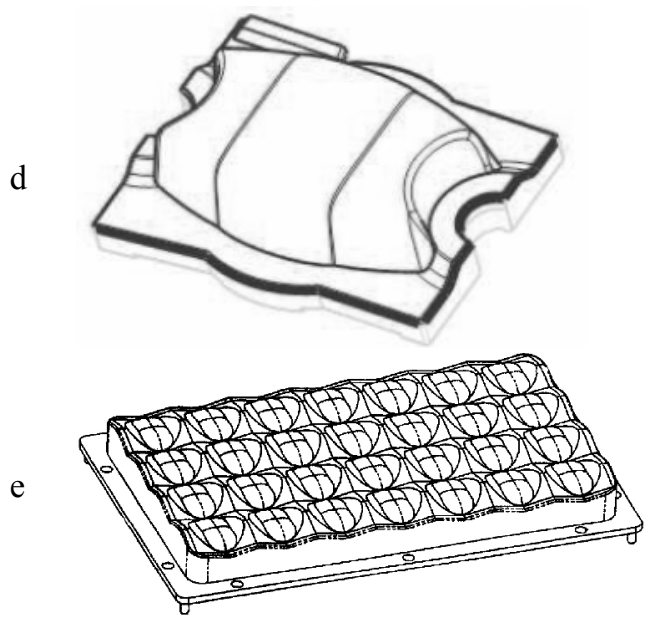

Fig. 9. Types of lenses for modifying LED luminous intensity distribution curve.
It should be mentioned about design drawbacks based on special elements of secondary optics:

- significant optical losses provided by several optical interfaces. Ideally, there should be only two optical interfaces: light-emitting diode - environment;

- requirement of positioning and aligning a great number of lenses relative to light-emitting diodes, that has a negative effect on the cost of LED street lights.

More reasonable way of getting the required luminous intensity distribution curve of LED lights is the use of LEDs with special primary optics instead of secondary ones. The best known example of such kind of LEDs is Golden Dragon Oval+ of Osram Opto company.

\subsection{Electrical design}

Electronics (power supply unit) is the next subsystem of LED street light. The main requirements to power supply unit of LED street lights are high efficiency $\eta_{\mathrm{el}}$, a more extensive range of operating voltages, in-built functions of overheat and overvoltage protection, galvanic isolation, high reliability (term of service), dust-proofing, moisture protection and lightning guard.

For this purpose in most cases LED street lights have power supply units of MeanWell, Moso, Inventronics and other companies. There is a tendency to use external power supply units in place of in-built units, as well as microprocessor control of LED street lights.

The advantage of LED street lights having an external power supply unit is that because of its less reliability in comparison with light-emitting diodes, it is installed separately from LED modules. Such installation of this power supply unit also makes it easy to repair it. Besides, the heat design of the luminaire is simplified due to removal of additional heat source from its package. At the same time, accessibility of power supply units at installation places makes great demands of electrical safety and anti-vandal implementation.

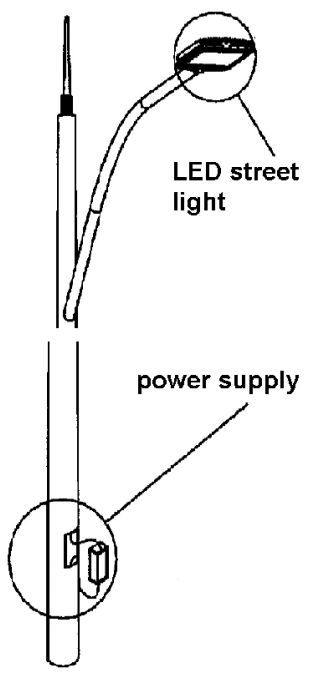

Fig. 10. Version of external power supply unit set for LED street light. 
As for microprocessor control of the luminaire, it provides light intensity monitoring of LED street light, depending on level of outside illuminance, season and current time of the day, as well as external signals, for example from remote controller by means of GSM, Internet $\left[{ }^{4}\right]$ or voice signals.

Besides, if LED street light is equipped with solar battery, the power supply unit would also provide optimazation of accumulator charging/discharging processes.

\section{Our results}

In conclusion, we shall review the results of our own elaborations in the area of LED illumination engineering for outside application.

One of the first projects in the world (2003-2004) on LED lighting of the pedestrian crossing is shown in Fig. 11. In this project, LED modules were placed under different angles (see Fig. 7 as well) to each other in order to have an oval-shaped light spot. LED device consisted of a linear luminaire and the pedestrian crossing sign with animation effect, set over the roadway on 5-meter height. Having power consumption not more than $60 \mathrm{~W}$, a linear luminaire provided maximum illuminance not less than 200 lux at 5-meter distance. The linear luminaire package was made from anodized aluminum, and shielding glass of polycarbonate. The device was characterized by in-built electronic block, providing the automatic switch on the light when outside illuminance dimmed below specified level.
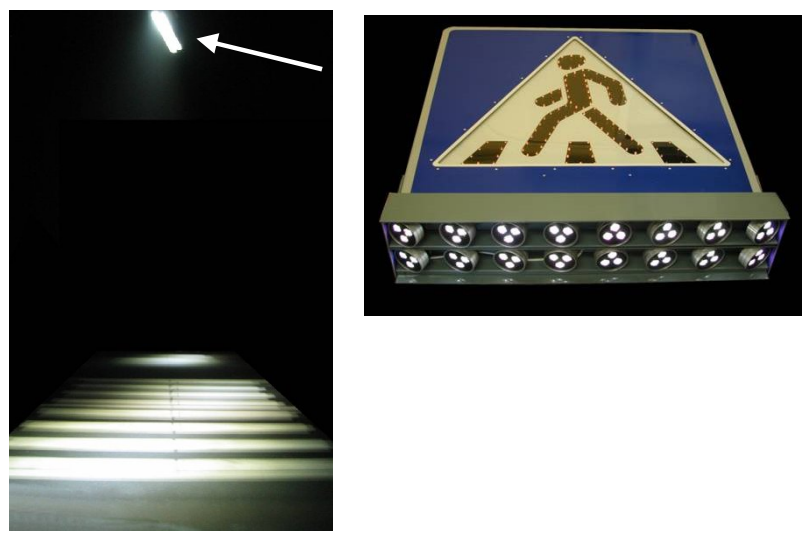

Fig. 11. LED lighting of the pedestrian crossing.

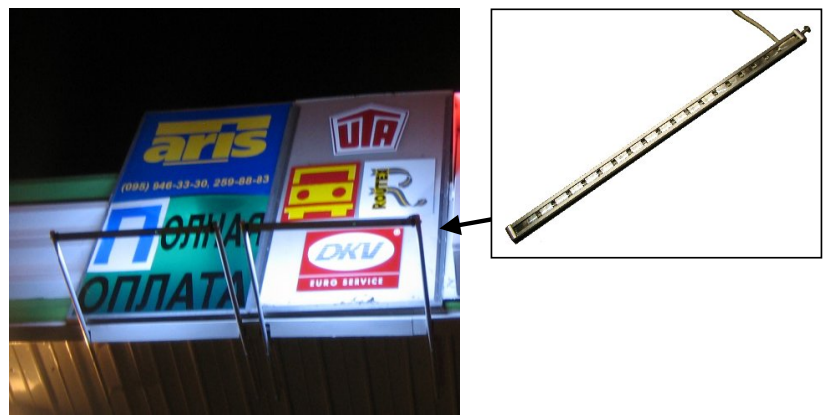

Fig. 12. LED lights for lighting of advertizing billboards.
There is another LED device designed for outside application. Fig. 12 shows LED luminaire for lighting of advertizing billboards.

The power of linear luminaire is $12 \mathrm{~W}$, and the axial illuminance at $1-\mathrm{m}$ distance is not less than 100 lux. To reach high illuminating engineering and operating characteristics, this luminaire includes $\mathrm{H}$ shaped reflectors with low optical loss factor and a special optically transparent UV-stabilized compound provided protection class IP 64, which are applied alongside with the use of effective light-emitting diodes. Power supply of the luminaire is provided by secondary source $24 \mathrm{~W} \pm 1 \%$ (up to 4 luminaries for one power supply unit).

\section{Conclusion}

Thus, it is necessary to optimize the thermal, optical and electrical subsystems of the luminaire to increase the efficiency of LED street light. The prospective optimization area of the thermal subsystem of the luminaire is the use of in-built heat pipes and ventilated packages. To optimize the optical subsystem of the luminaire, different methods can be used, but in our opinion, the most promising way is to use LEDs with special primary optics or engineering solutions having minimum quantity of optical interfaces. In order to increase reliability and efficiency of electrical subsystem of the luminaire, it is reasonable to set power supply units separately from LED modules. This enables to improve the heat design and increase the repairability of luminaries.

Besides, taking into consideration physiological features of human eyesight (scotopic and photopic ones) as well as dependence of light intensity perception on correlated color temperature, it is reasonable to lower requirements in relation to the level of illuminance for LED street lights.

\section{References}

1. www.energo.by.

2. Yu.V. Trofimov, S.I. Lisnik, V.I. Tsvirko, V.S. Posedko, V.V. Doctarau, V.V. Maziuk, Using Heat Pipes in LED Lights. Semiconductor Lasers and Systems on Its Base / Eds V.Z. Zubelevich, V.K. Lononenko, G.P. Yablonskii // VII Belarussian-Russian Seminar, Minsk, IPh NAS of Belarus, 2009, p. 141-144.

3. K.L. Gordon et al., Spectrally Enchanced Lighting Program Implementation for Energy Savings: Field Evaluation. Pasific Northwest National Laboratory, U.S. Department of Energy, 2006, p. 118.

4. www.betaled.com.

5. www.lioris.eu. 